\title{
The Salmon of Knowledge
}

\author{
Scott A. Akenhead ${ }^{1}$, George Batten ${ }^{2}$, Tom Bird ${ }^{3}$, Nghia Doan ${ }^{4}$, James R. Irvine ${ }^{1}$, Oksana Korol ${ }^{5}$, Chantal \\ Nessman $^{1}$, and Peter O'Blenis ${ }^{6}$ \\ ${ }^{1}$ Fisheries and Oceans Canada, Pacific Biological Station, 3190 Hammond Bay Road, Nanaimo, BC, Canada \\ ${ }^{2}$ Sitka Technology Group, \#229-525 $3{ }^{\text {rd }}$ Street, Lake Oswego, OR, 97034, USA \\ ${ }^{3}$ Fisheries and Oceans Canada, Northwest Atlantic Fisheries Centre, 80 East White Hills Rd, St. John's, Canada \\ ${ }^{4}$ Agiga Quanta Inc., 42 Amberly Place, Ottawa, ON, K1J 8A1, Canada \\ ${ }^{5}$ Fisheries and Oceans Canada, 200 Kent St, Ottawa, ON, K1A 0E6, Canada \\ ${ }^{6}$ Fisheries and Oceans Canada, Bedford Institute of Oceanography, Challenger Drive, Dartmouth, NS, Canada
}

Keywords: knowledge graph, information technology, program management, decision support, salmon restoration, natural language processing

We developed a knowledge management system for salmon managers that is capable of dealing with the complexity of multi-agency salmon fisheries and habitat management programs, despite the dynamic nature and sheer volume of information involved. This enterprise IT system was designed, installed, loaded, and exercised by Fisheries and Oceans Canada (DFO). Features include advanced security, automated data-processing, and an extensible user-friendly interface.

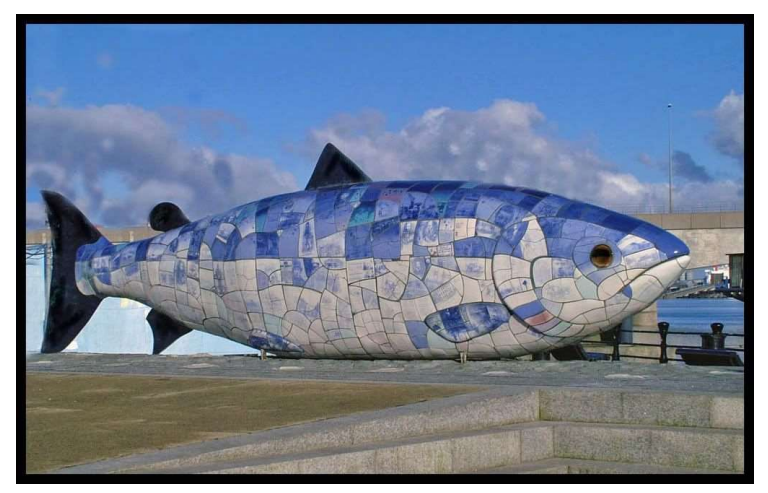

Fig. 1. The Salmon of Knowledge is a large statue in Belfast, Ireland, that celebrates an ancient myth and the 1991 return of salmon to River Lagan after being absent since 1774.

The Salmon of Knowledge (Fig. 1) is a huge statue in Belfast that commemorates an ancient Irish myth. Each scale on the statue represents some of the knowledge required to re-establish Atlantic Salmon in River Lagan in 1991. The myth describes a magic salmon that possessed all of the knowledge in the world and how the first person to taste it would acquire that knowledge. Without spoiling a good story, let us take that magic as a metaphor ${ }^{1}$ to make us think about building and managing our knowledge about salmon, specifically in the context of the International Year of the Salmon (IYS).

IYS has an overarching goal for the future: Salmon are resilient. This means salmon populations can survive surprise from careless industrialization and global warming, in addition to unpredictable, natural, ecosystem dynamics. IYS elaborates: "to realize a shared future with salmon, an effort beyond the capacity of any one group or country is needed to raise awareness, address knowledge gaps, and devise new approaches to informing and taking decisions." This echoes Holling (1973):

"A management approach based on resilience... would emphasize the need to keep options open, the need to view events in a regional rather than a local context, and the need to emphasize heterogeneity. Flowing from this would be not the presumption of sufficient knowledge, but the recognition of our ignorance; not the assumption that future events are expected, but that they will be unexpected. The resilience framework can accommodate this shift of perspective, for it does not require a precise capacity to predict the future,

${ }^{1}$ Any sufficiently developed technology is indistinguishable from magic (Clarke 1968).

All correspondence should be addressed to S. Akenhead. 
but only a qualitative capacity to devise systems that can absorb and accommodate future events in whatever unexpected form they may take."

Starting in 2017, we addressed this problem, devise resilience, from the perspective of knowledge management, collaboration, and decision support (Fig. 2). The problem posed to us by DFO in 2019 was knowledge assembly for a scenarios-based, fisheries-management decision: efficient discovery of who knows what we need to know to make a better decision? Note two meanings: (1) finding metrics for prescribed decision criteria that will be used to compare scenarios; and (2) discovering unrealized but important decision criteria that should be added to the decision process.

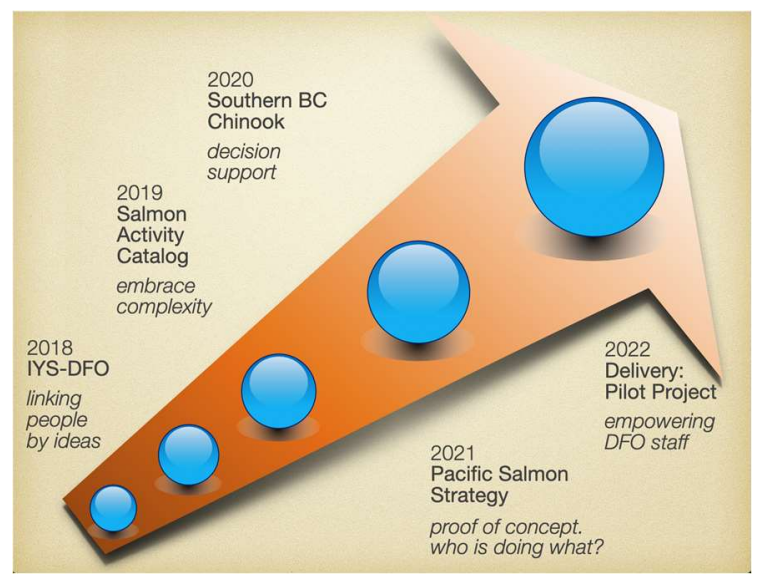

Fig. 2. A series of experiments and prototypes led to the existing system for the salmon knowledge graph.

We hypothesize that part of the decision-support problem stems from how information is stored, managed, and inter-related within and between knowledge sources. The information sources in use today are lists and tables, structures which assume that knowledge units are static in form and relatively harmonized in terms of their structure - an approach that rapidly falls apart without a rigorous, top-down, structure for knowledge management. Because of the large number of heterogeneous information sources involved, our approach was to use graph database technology (neo4j.com) to cope with:

(a) the scope, diversity, and complexity of salmon ecosystems, including all aspects of human impacts and interests;

(b) the fluidity of projects, agencies, habitats, problems, and methods - both the content and the structure of salmon information are dynamic;

(c) the need for provenance of datasets and analysis results, thus defensible decisions;

(d) information discovery by following chains of indirect links; and

(e) epistemology: using a rich set of ideas to organize, navigate, and apply salmon information.

Essentially, this technology is more similar to how humans think, compared to a relational database. The ideas tabled in the NPAFC/IYS workshop International Salmon Data Laboratory (Akenhead et al. 2019) accelerated our thinking.

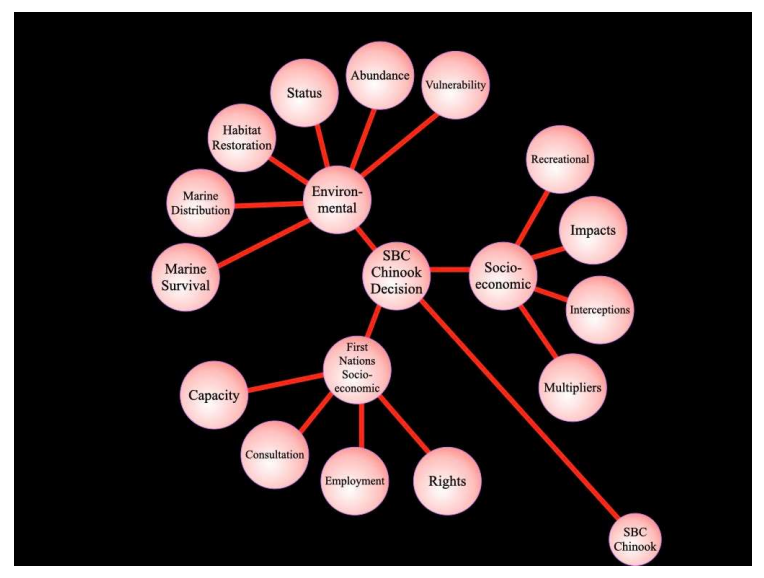

Fig. 3. The Salmon Knowledge Graph (2020) used Idea nodes to organize, discover, and apply the knowledge required for decisions. Ideas included taxonomy: Southern BC Chinook; general decision criteria: Environmental, First Nations; and specific decision metrics: Employment, Stock Status. 
By March 2020 we were able to demonstrate how a salmon knowledge graph could manage the ideas involved in decision support (Fig. 3) as a means of organizing and discovering the knowledge required for fully informed and fully inclusive decisions.

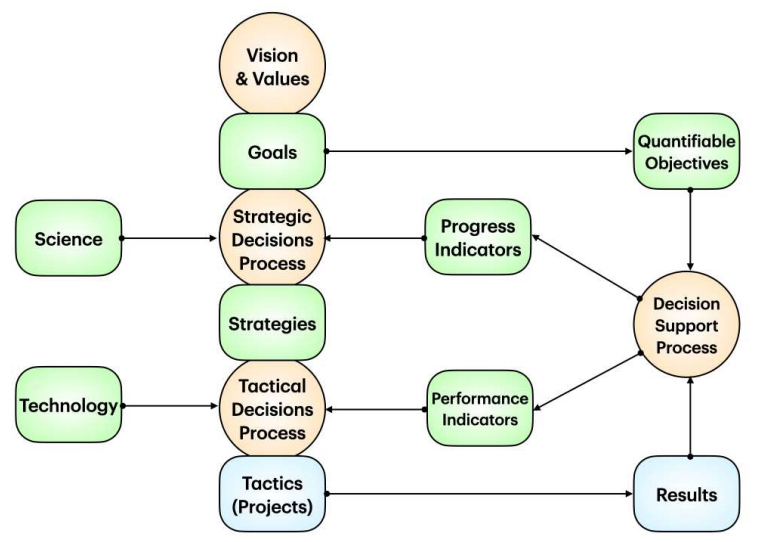

Fig. 4. Adaptive management applies learning from experience (outcomes monitoring) to replace elements of a strategic plan. Enabling those replacements, available from science and technology, requires a decision process embedded in the strategic plan. Managing such a process - across many salmon-related agencies, programs, projects, and people — can be enabled by a knowledge graph.

A related problem was posed in 2021: program management for many salmon restoration projects conducted by many agencies in DFO's Pacific Region. Our perspective on this problem is Adaptive Management (Holling 1978, Gunderson and Holling 2002; but see Walters 2007), which we see as convolving strategic planning and decision support (Fig. 4). Problems thwarting efficacious, multi-agency, program management include:

- too much information: 100,000s of projects, people, places, agencies, documents, datasets;

- too complicated: many dimensions, each diverse: salmons' lives; habitat metrics; observation and analysis methods; funding and management; economic, social, and cultural impacts;

- too dynamic: churn of participants, obsolescence of information, crippling lags before updates;

- too hidden: information is buried in technical reports, web pages, scattered, ad hoc, Excel files;

- too isolated: important connections are missing, information is simply not shared.

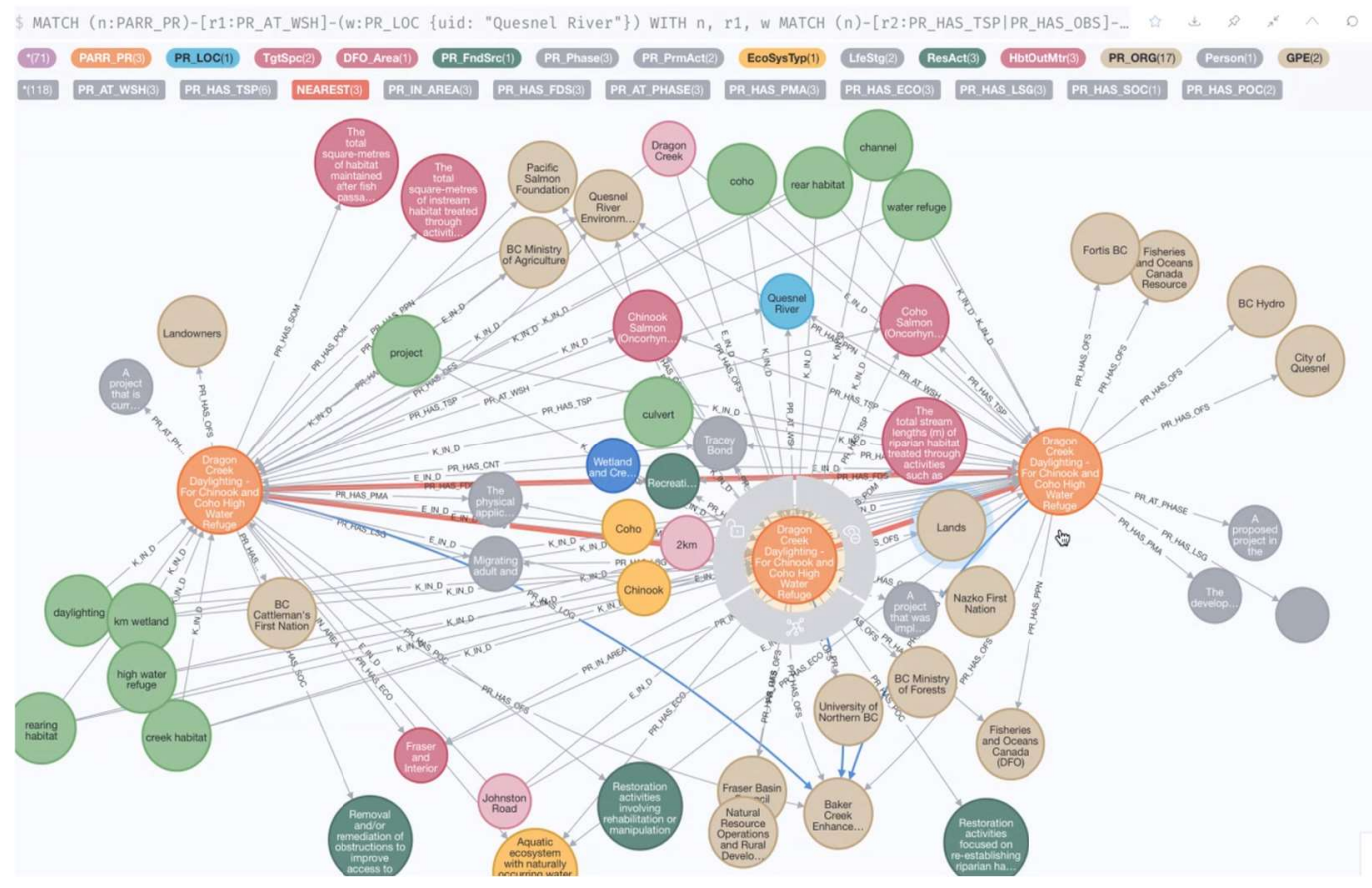

Fig. 5. A query (Cypher, via Neo4j Browser) to display everything in the graph linked to salmon restoration projects linked to the Quesnel River Watershed. A user can review the information within a node and expand further links from a node to discover unrealized connections: new knowledge. 
The 2021 project proceeded with an emphasis on tools and technology. We received a dataset (MS Excel) containing metadata for $>800$ salmon restoration projects. This was converted to a knowledge graph by a combination of (a) code in Python and Cypher to dissect complicated Excel data into nodes and links; (b) natural language processing to extract key phrases and entities (Person, Place,); (c) base layers( 180,000 [total] of place names, postal codes, and First Nations agencies were loaded); and (d) artificial intelligence to automatically generate links such as "project has place,", "agency has place,". The result, merely a Proof of Concept so far, was an unprecedented ability to visualize and traverse information in a process of knowledge discovery (Fig. 5).

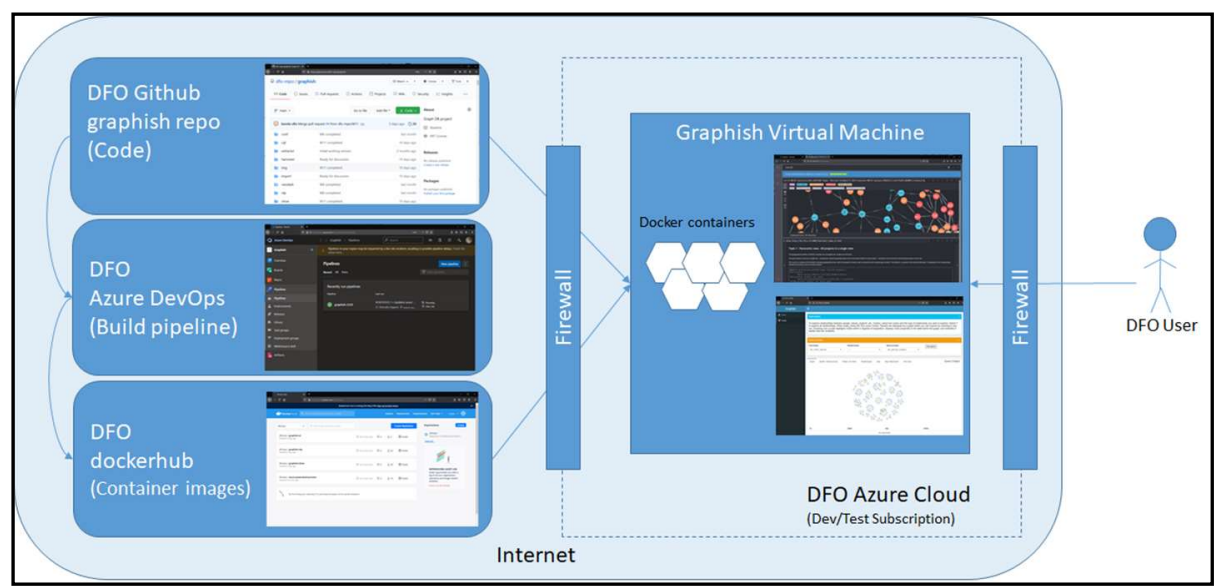

Fig. 6. The knowledge graph system implemented by Fisheries and Oceans Canada was designed as cloud-based enterprise IT with appropriate cyber-security and CD/CI tools (continuous development, continuous integration). This system, Graphish, can be re-implemented quickly and safely by other agencies. Less demanding installations, including personal computers, will not incur license costs.

Connecting automated data-processing pipelines to the knowledge graph was an important achievement, stemming from the data extraction, loading and linking process. These pipelines promise radical efficiency in the near future, for updating the knowledge graph and delivering analyses including decision indicators. After initial coding, these pipelines are available to users as autonomous containers (Docker) held in a cloud-based, enterprise IT system (MS Azure, DevOps), as designed and implemented by DFO (Fig. 6). Rigorous attention to cyber-security was exacted. The Salmon Knowledge Graph is just one application of this system (Graphish); knowledge graphs for other communities of practice can be instantiated quickly and the technical design can be replicated safely by other organizations.

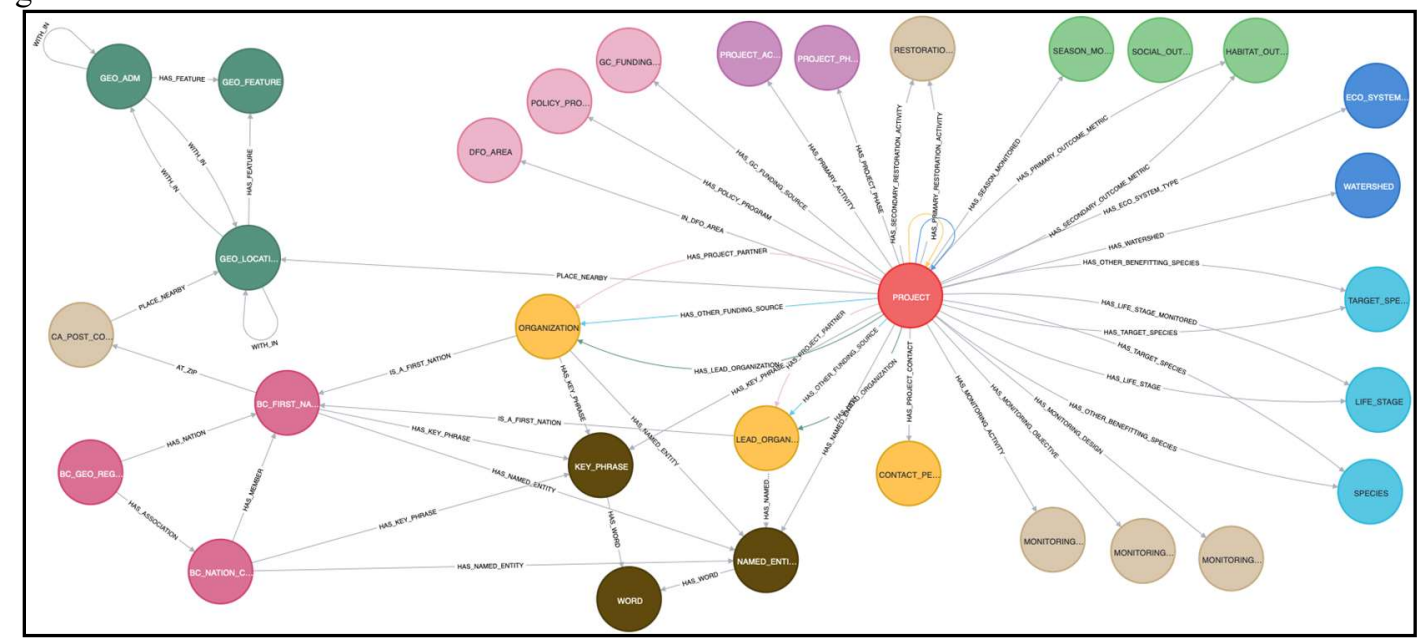

Fig. 7. The salmon knowledge graph schema was derived from a dataset of salmon restoration projects.

Automated creation of links between nodes related to program management was facilitated by base layers of (left side) places in GeoNames.org, postal codes, and First Nations; and (center bottom) key phrases extracted by natural language processing.

The schema for the 2021 salmon knowledge graph (Fig. 7) was derived from the reported structure of the salmon restoration projects. This was an enlightening change in perspective from the preceding top-down design 
derived from ideas about decisions and planning. The schema (labels that index many instances of nodes and links) is the basis for navigating and applying the knowledge graph.

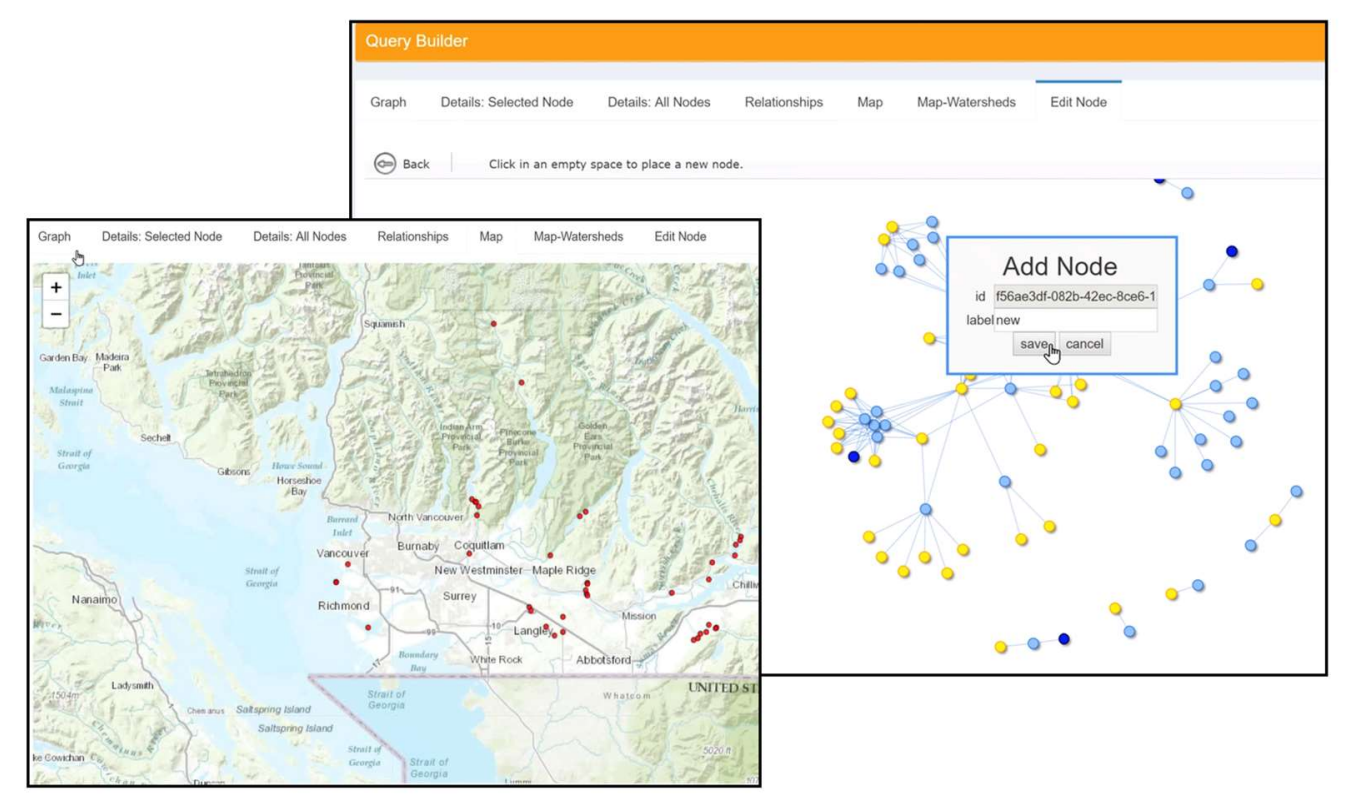

Fig. 8. The prototype user interface allows exploration of the salmon knowledge graph by interactive Views, currently including maps, tables, and networks (graphs). Queries are constructed by picking from lists of node and link types and instances. Users who are not programmers can add and edit information with this interface, implying on-going, community-wide, improvement. New Views, that control and present automated data processing, are easily added as a means to new efficiencies. A four-minute demonstration: (https://drive.google.com/file/d/1jbDT-4r4CPmJ9URb5Vzof6_KEzPSc00g).

By March 2021 we were able to demonstrate additional value from this technology in two ways:

1. We showed that sophisticated and penetrating questions, difficult to pose to a relational database (sequential joins), could be expressed quickly as knowledge graph queries. Examples include: changes in patterns of project ownership over time; identifying First Nations with interests in a watershed (spatial analysis) that are not linked to salmon restoration projects in that watershed; and

2. Skill barriers were lowered by user-friendly web forms that enabled information discovery and reports. This user interface (Graphishui, via R and Shiny) presents various Views of the information (Fig. 8) such as GraphView (network) for both discovering and editing information, and MapView for realizing spatial patterns. This interface is a software platform that will support many further Views, each as a tool that might involve automatic or semiautomatic data processing, each delivering value from salmon knowledge management.

\section{REFERENCES}

Akenhead, S.A., N. Bendriem, and J. Park (Editors). 2019. Report of the proceedings for the IYS workshop: First International Year of the Salmon Data Laboratory (ISDL) Workshop. N. Pac. Anadr. Fish Comm. Tech. Rep. 14. 31pp. (Available at https://npafc.org)

Clarke, A.C. 1968. Clarke's third law on UFO's. Science 159 (3812). pp. 255. doi: 10.1126/science.159.3812.255-b.

Gunderson, L., and C.S. Holling (Editors). 2002. Panarchy: Understanding transformations in human and natural systems. Island Press, Washington. $450 \mathrm{pp}$.

Holling, C.S. 1973. Resilience and stability of ecological systems. Annu. Rev. Ecol. Syst. 4:1-23.

Holling, C.S. (Editor). 1978. Adaptive environmental assessment and management. John Wiley, New York. 377 $\mathrm{pp}$.

Walters, C.J. 2007. Is adaptive management helping to solve fisheries problems? Ambio 36(4): 304-307. 